\title{
Molecular analysis of hemagglutinin, neuraminidase, matrix genes provide insight into the genetic diversity of seasonal H3N2 human influenza a viruses in Bangladesh during July-August, 2012
}

\author{
Mukesh Jain $^{1}$ - Sohidul Islam ${ }^{1}$ - A. S. M. Zisanur Rahman ${ }^{1}$ Sharmin Akhtar ${ }^{1}$. \\ Kazi Nadim Hasan ${ }^{1}$ - Gias Uddin Ahsan ${ }^{2,3}$ - Abdul Khaleque ${ }^{1} \cdot$ Maqsud Hossain $^{1,3}$
}

Received: 15 October 2017/ Accepted: 20 January 2018/Published online: 1 February 2018

(C) Indian Virological Society 2018

\begin{abstract}
Influenza A virus subtype $\mathrm{H} 3$ is a threat to public health and it is important to understand the evolution of the viruses for the surveillance and the selection of vaccine strains. Comparative analysis of four Bangladeshi isolates with isolates circulating other parts of the world based on three candidate genes hemagglutinin (HA), neuraminidase (NA), matrix protein (MA) showed no evidence of significant distinct subclade of viruses circulating in the country over the period of study. Despite these findings, we found $\mathrm{N} 161 \mathrm{~S}$ substitution in all four $\mathrm{H} 3 \mathrm{~N} 2$ influenza stains resulting in the gain of NSS160-162 glycosylation site. All H3N2 Influenza subtypes in the study had amino acid substitution at position 31 on the M2 protein (Aspartic acid to Asparagine) which is known to be responsible for amantadine drug resistance.
\end{abstract}

Keywords H3N2 - Hemagglutinin · Neuraminidase · Matrix genes

Electronic supplementary material The online version of this article (https://doi.org/10.1007/s13337-018-0431-y) contains supplementary material, which is available to authorized users.

Abdul Khaleque

abdul.khaleque@northsouth.edu

$\triangle$ Maqsud Hossain

muhammad.maqsud@northsouth.edu

1 Department of Biochemistry and Microbiology, North South University, Dhaka 1229, Bangladesh

2 Department of Public Health, North South University, Dhaka 1229, Bangladesh

3 NSU Genome Research Institute (NGRI), North South University, Dhaka 1229, Bangladesh

\section{Introduction}

Influenza viruses are enveloped viruses belong to the family Orthomyxoviridae consist of negative-sense RNA genome [14], which can cause highly contagious respiratory illness. According to World Health Organization (WHO), influenza epidemics are responsible for three to five million cases of severe illness and 250,000-500,000 deaths annually worldwide (http://www.who.int/iris/han dle/10665/68985). In temperate regions, influenza epidemics occur seasonally and peak in the winter and are almost absent in the summer. The influenza pandemic were reported three times during the 20th century 1918, 1957 and 1968 (http://www.who.int/mediacentre/factsheets/ fs211/en/). The 1918 pandemic had the highest mortality, causing approximately 40 million deaths worldwide, with an unprecedented number of deaths of young adults [16]. The virus is classified into subtypes $\mathrm{H} 1$ to $\mathrm{H} 18$ and $\mathrm{N} 1$ to N11 by previously reported of eight hemagglutinin (HA) and eleven neuraminidase (NA) types [22]. An important ability of influenza A viruses is re-assortment which means that two or more viruses infecting the same cell can recombine their genetic segments to form a new virus strain [2]. By re-assortment, influenza A viruses achieve genetic shift, rapid changes of viral properties that can give rise to a new strain unrecognized by host immune defense and thus capable of pandemic spread. By point mutations in immunogenic parts of the genome, influenza A viruses also makes much more gradual changes termed genetic drift, these changes allow the viruses to escape some host immunity and to cause seasonal influenza outbreaks. According to the WHO report (27 July 2015) in tropical Asia including those in Southern Asia the influenza subtype A (H3N2) predominate with co-circulation of influenza type A (H3N2) and B. However, there is no systematic 
study in Bangladesh on molecular characterization of this Influenza virus A. Since these viruses are highly variable and changing rapidly this pilot study was undertaken to characterize four $\mathrm{H} 3 \mathrm{~N} 2$ isolates and this will form the basis of understanding of precaution against new H3N2 strains.

\section{Materials and methods}

\section{Sample collection and viral culture}

A total of four Influenza A positive nasopharyngeal swab samples were collected from Dhaka Medical College and Hospital between July and August, 2012. After obtaining signed informed consent from the hospital authority, we collected throat and nasal swab samples from patients those are previously confirmed as influenza positive influenza like illness (ILI), defined as subjective fever and (cough or sore throat) by senior physician. We also collected samples from the patients admitted in the medicine and pediatrics inpatient departments who met the case definition of severe acute respiratory illness (SARI), defined as fever $\left(>38^{\circ} \mathrm{C}\right)$ and (cough or sore throat) and (shortness of breath or difficulty breathing). At a time of sample collection Influenza positive patients were managed with supportive care and no antiviral drug such as tamiflu was given.

The clinical samples were cultured by tissue culture technique using the MDCK.2 (ATCC; CRL-2936) cellline. Virus culturing was performed using trypsin treated MDCK according to CLSI Viral Culture; Approved Guideline [4]. Influenza A/H3N2 positive samples were stored for further analysis.

\section{RNA extraction and sequencing}

Viral RNAs were extracted from the culture supernatants using a QIAmp Viral RNA Mini Kit (QIAGEN, Hilden, Germany). Three RNA gene segments (HA, NA, M) were reverse transcribed using Biometra T1000 PCR cycler (Biometra, Germany) following the recommended protocol [10]. Following the reverse transcription three partially gene segments were amplified using specific primers

(HA F: TGTAAAACGACGGCCAGTAAGCAAAAGC AGGGGAAAATA,

HA R: CAGGAAACAGCTATGACCTAGTAGAAA CAAGGGTGTTTT-1778 bp;

NA F: TGTAAAACGACGGCCAGTAAGCAAAAG CAGGAGTGAAAA,

NA R: CAGGAAACAGCTATGACCAGTAGAAA CAAGGAGTTTTTT-1413 bp;

M F: TGTAAAACGACGGCCAGTAGCAAAAGCAG GTAG,
M R: CAGGAAACAGCTATGACCGCAATCTGYTCACAKGT-474 bp) and QIAgenTaq DNA polymerase (Germany). DNA sequencing was performed at DNA Solution, Dhaka, Bangladesh by the dideoxynucleotide chain termination method with an ABI PRISM Dye Termination Cycle Sequencing Ready Reaction kit in an ABI PRISM automated sequencer (Perkin-ElmerApplied Biosystems, Foster City, Calif).

\section{Sequence analysis}

DNA sequence editing and analysis were performed with Chromas 2.3 (Technelysium, Helensvale, Australia). Sequences are available at GenBank under the accession numbers KY801317-KY801328. Multiple sequence alignment was performed using ClustalW in Bioedit, version 7.1.3 (http://www.mbio.ncsu.edu/bioedit/page2.html). Phylogenetic and molecular evolutionary analyses were conducted using the MEGA version 5.0 software package [20]. Genetic distances were calculated using the Kimura 2-Parameter method [10]. The dendrograms was constructed using the neighbor-joining method [18]. The antigenic site comparison was carried out by BioEdit sequence alignment editor. All the three sequences were submitted to program (http://www.ncbi.nlm.nih.gov/ BLAST/) to identify the similar sequences. Potential $\mathrm{N}$ glycosylation sites were predicted using Net NGlyc 1.0 server [21]. The 3D structures of HA and NA proteins for Bangladeshi strains were modeled; HA and NA variation sites were labeled in the 3D structures by $\mathrm{Cn} 3 \mathrm{D}$ software [25].

\section{Results}

\section{PCR and comparative analysis of HA, NA and M genes}

A total of four influenza viruses $\mathrm{A} / \mathrm{H} 3 \mathrm{~N} 2$ samples were collected in 2012 from Dhaka Medical College in Bangladesh. To study the genetic characteristics of the human H3N2 viruses circulating in the country and to investigate genetic diversity, partial sequences of three segments of A/H3N2 influenza genome was performed. Initially RNA was extracted from tissue cultured samples using QIAmp Viral RNA Mini Kit (QIAGEN, Hilden, Germany) according to the manufacturer's instruction. Following the conversion of RNA to cDNA by reverse transcriptase, amplification was done using specific primers for each segment. The PCR result was observed by gel electrophoresis which showed band at position 1778, 1413, and 474 bp representing HA, NA and M genes, respectively. 
For comparative analysis of the sequences of HA, NA and M segments of Influenza A/H3N2 viruses we used 37 global isolates from GenBank and GSAID as reference strain from each year pandemics and isolates from 1968 to 2012. Bangladeshi $\mathrm{A} / \mathrm{H} 3 \mathrm{~N} 2$ isolated under study showed at least $>97 \%$ similarity with strains all over the world: Asia, Europe, Australia, Africa and North and South America.

\section{Hemagglutinin (HA) segment analysis}

A similarity matrix was constructed using 'Poisson correction' model showing similarity percentages of amino acid sequence of global and Bangladesh isolates of influenza A/H3N2 virus HA segment (Supplementary File 1).

The comparison revealed that the HA segment of our strains were identical with Washington (A/Washington/20/ 2012) and Singapore (A/Singapore/H2011.570/2011) strains. These strains also showed more than $99.6 \%$ similarity with vaccine strains (A/Victoria/361/2011) from northern hemisphere. Four major clusters can be found in phylogenetic tree (Supplementary Figure 1): Group I showing the circulating isolates of H3N2 from 2005 to 2012 across the world, Group II, III and IV showing the clustering of $\mathrm{H} 3 \mathrm{~N} 2$ isolates before 2003. It was interesting to note that isolates clustered based on their year of isolation rather than their geographical location of isolation. The phylogenetic tree of HA gene showed that isolates circulating in 2012 season in Bangladesh clustered in Group I with the other isolates from rest of the world of 2009, 2010, 2011 and 2012. The Bangladeshi isolates were closely related to isolates from Vaccine strain isolated from Moscow, Singapore, Japan, and Korea on the year of 2012. Isolates from other groups showed various clades of $60 \mathrm{~s}$, $70 \mathrm{~s}, 80 \mathrm{~s}, 90 \mathrm{~s}$ and $21 \mathrm{st}$ century. The analysis showed that the HA sequences were at the same branch with the vaccine strains and there was no evidence of a significant distinct subclade of viruses circulating in Bangladesh.

\section{Neuraminidase (NA) segment analysis}

The comparison revealed that the NA segment of our strains was very closely related to a Japanese strain

(A/Kyoto/11K005/2012 \& A/Osaka/11K114/2012) of 2012 isolate with more than $99.7 \%$ identity (Supplementary File 2). Nevertheless, it showed more than $99.0 \%$ similarity with the WHO recommended vaccine strain (A/ Victoria/361/2011) of northern hemisphere. Apart from these similarities it also showed more than $99.5 \%$ similarity with the Singapore stain (A/Singapore/H2011.797/2011). Beside these findings an America strain (A/California/35/ 2012) of 2012 showed more than $99.5 \%$ similarity with Bangladeshi strains. However, comparison of with the sequences of NA segments with vaccine strain and isolates from other continents including Asia (China, Japan, Singapore), Russia, USA of the world from 1968 to 2012, only Bangladeshi strain reside with vaccine strain with the maximum similarity. Phylogenetic analysis for NA gene resulted in three major clusters (Supplementary Figure 2) and less heterogeneous compared to the phylogeny of HA gene (Supplementary Figure 1). Interestingly isolated grouped into different clusters based on the time they were isolated: Group I includes the isolates from 2007 to 2012, whereas, Group II and Group III from 1968 to 2003 isolates. Analysis of the NA gene segment revealed the same pattern as the phylogenetic tree of HA segment. The NA sequence of the Bangladesh fell into the corresponding clade and were similar to those circulating in these years and there was no evidence for the emergence of a significant distinct subclade (Supplementary Figure 2). The representative Bangladeshi isolate of 2012 reside with WHO recommended vaccine strain and clustered in Group I with closest relationship with those viruses sampled in 2011 and 2012.

\section{Matrix protein (MA) segment analysis}

The $\mathrm{M}$ segment of Bangladesh strains was identical to a USA strains (A/Michigan/03/2012, A/NewYork/16/2012) and another Singaporean (A/Singapore/H2011.499a/2011) strain (Supplementary File 3) and it showed 100\% similarity with the WHO recommended vaccine strain (A/ Victoria/361/2011) from northern hemisphere as well as another Australian strain (A/Australia/55/2009) of 2009. Although phylogenetic analysis of $\mathrm{M}$ gene shows more diversity among isolates from different years resulting in five major groups Group I includes isolates from 2007 to 2012, and others with the isolates from 1968 to 1994 . It was found that the strain of Bangladesh (2012) including others circulating in the similar period clustered in Group I, however, the WHO recommended vaccine strain for Northern Hemisphere A/Victoria/361/2011 (Supplementary Figure 3) and the vaccine strains were found more diverse compared to the HA and NA phylogeny.

\section{Analysis of glycosylation sites, antigenic sites and drug resistance}

We identified 10 potential glycosylation sites in the HA gene of all the Bangladeshi isolates of 2012 with a threshold value of < 0.5: NST024-026, NGT038-040, NCT079-081, NWT142-144, NGT149-151, NSS160-162, NVT181-183, NST262-264, NGS301-303 and NGT499-501. For the/ Victoria/361/2011

HA gene, 10 potential glycosylation sites were present, with only NSS160-162 missing. Using the A/Victoria/361/ 2011 HA gene as a reference, N161S was seen in 2012 


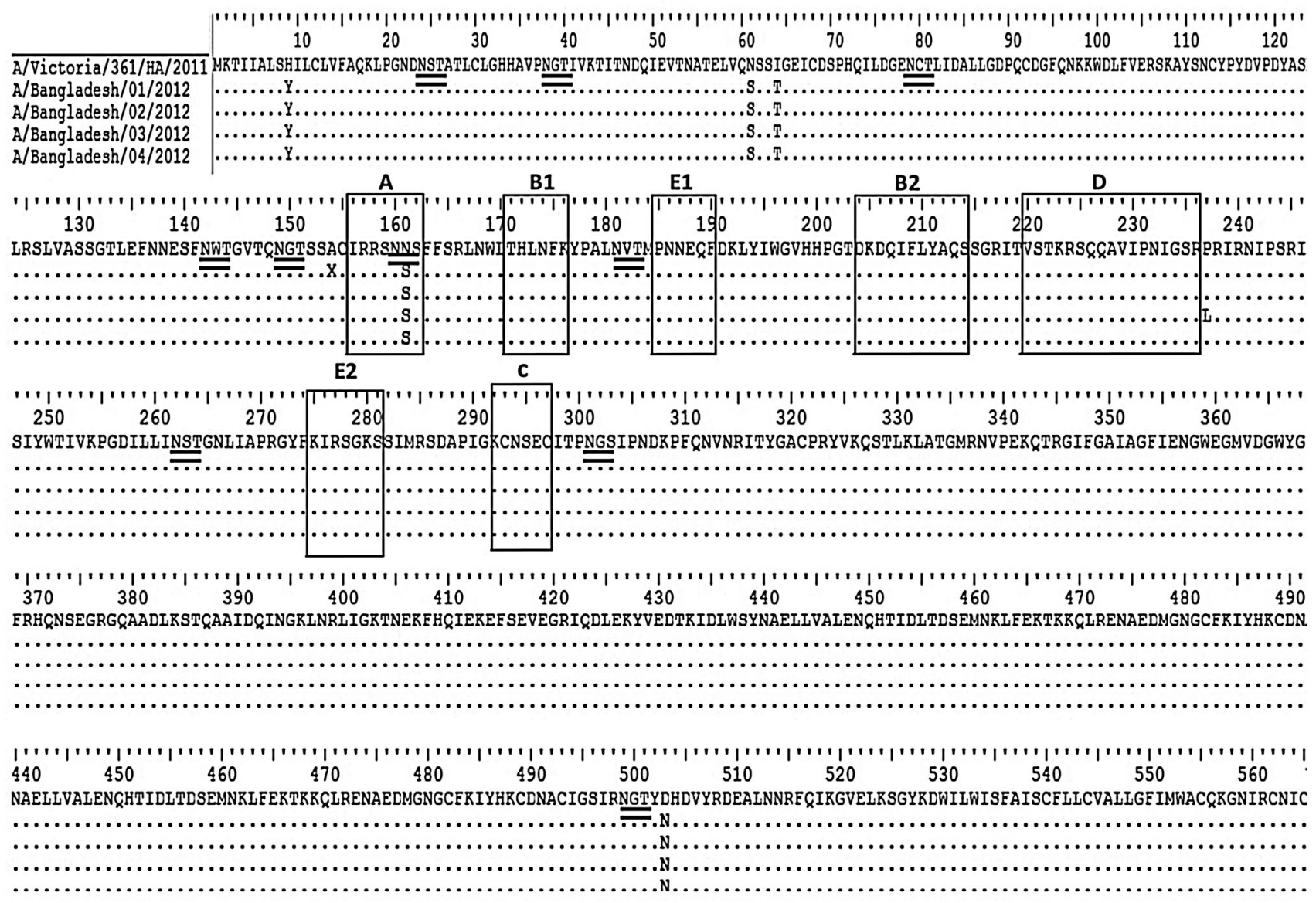

Fig. 1 Amino acid comparison between HA domains of H3N2 Bangladeshi isolates and vaccine strains. Dots represent amino acids similar to the consensus. The amino acid residues mapped at

Bangladeshi strains, resulting in a gain of the NSS160-162 glycosylation site on the other hand N61S resulting in an deletion of the NSS60-62 glycosylation sites. Some amino acids are specific determinants of antigenic recognition of HA protein by specific antibodies. They constitute five distinct regions called epitopes A-E [23, 27] with some separated regions, i.e., B1, B2, E1, and E2. To evaluate the impact of genetic changes in influenza A (H3N2) viruses detected in Bangladesh on immune recognition, an alignment of the epitope regions of four amino acid sequences was performed together with the $\mathrm{A} / \mathrm{Victoria} / 361 / 2011$ virus (Fig. 1). The comparison revealed that all Bangladeshi strains had a serine instead of asparagine residue at position 161 (N161S), which modified a glycosylation site by polar amino acid to tiny polar amino acid in antigenic site A, similar to the previous studies where K161N substitution transpired [3, 8, 15]. K161N substitution is characteristic of viruses similar to $\mathrm{A} / \mathrm{California} / 7 / 2004$. This change was an exchange of a positive to a polar amino acid and there is some evidence that this might be responsible for an antigenic drift $[3,6,8,15]$. In comparison of nucleotide and previously defined antigenic sites A-E are shown as large rectangles. The potential $\mathrm{N}$-glycosylation sites, with threshold value of 0.5 , are double-underlined

deducted amino acid sequences of NA from these viruses, a high degree the other studies $[3,5,6,8,15]$ conserved residues for catalytic sites (R118, D151, R152, R224, E276, R292, R371 and Y406) and also framework sites supporting the catalytic residues (E119, R156, W178, D198, I222, E227, H274, E277, N294 and E425) were detected. The variable regions in the NA head domain are amino acids $140-155,328-370$ and to a lesser extent $381-403[2,7]$ and Bangladesh isolates also showed some changes corresponding to the vaccine strains are illustrated in the Table 1. The NA sequence of the Bangladeshi fell into the corresponding clade and were similar to those of equivalent viruses circulating in this years and there was no evidence for the emergence of a significant distinct subclade. Moreover, we used C3n software for create a 3D model of hemagglutinin, neuraminidase and matrix protein and shown the changes in 3D model and mutation sites compare with vaccine stain and finally edited manually (Fig. 2a-c).

Alterations at positions prone to induce drug resistance were observed. Oseltamivir resistant strains have been 
Table 1 Amino acid alteration between Bangladeshi isolates and vaccine strain

\begin{tabular}{|c|c|c|c|c|c|c|c|c|c|c|c|c|c|c|c|}
\hline \multirow[t]{2}{*}{ Bangladeshi isolates } & \multicolumn{7}{|c|}{ Hemagglutinin } & \multicolumn{8}{|c|}{ Neuraminidase } \\
\hline & $\mathrm{H} 9$ & N61 & I64 & A154 & N161 & P237 & D503 & G93 & $\mathrm{T} 148$ & $\mathrm{X} 151$ & T153 & K258 & T329 & D 356 & $\mathrm{X} 414$ \\
\hline A/Bangladesh/01/2012 & $\mathrm{Y}$ & $S$ & $\mathrm{~T}$ & $\mathrm{X}$ & $S$ & $\mathrm{P}$ & $\mathrm{N}$ & $\mathrm{D}$ & I & $\mathrm{D}$ & $\mathrm{T}$ & $\mathrm{E}$ & $\mathrm{N}$ & $\mathrm{D}$ & G \\
\hline A/Bangladesh/02/2012 & $\mathrm{Y}$ & $S$ & $\mathrm{~T}$ & A & $S$ & $\mathrm{P}$ & $\mathrm{N}$ & $\mathrm{D}$ & $\mathrm{X}$ & $\mathrm{D}$ & $X$ & $\mathrm{E}$ & $\mathrm{N}$ & A & G \\
\hline A/Bangladesh/03/2012 & $\mathrm{Y}$ & $S$ & $\mathrm{~T}$ & A & $S$ & $\mathrm{~L}$ & $\mathrm{~N}$ & $\mathrm{D}$ & $\mathrm{T}$ & $\mathrm{D}$ & $\mathrm{T}$ & $\mathrm{E}$ & $\mathrm{N}$ & $\mathrm{D}$ & G \\
\hline A/Bangladesh/04/2012 & Y & $\mathrm{S}$ & $\mathrm{T}$ & A & $\mathrm{S}$ & $\mathrm{P}$ & $\mathrm{N}$ & $\mathrm{D}$ & $\mathrm{T}$ & $X$ & $\mathrm{~T}$ & E & $\mathrm{N}$ & D & $\mathrm{G}$ \\
\hline
\end{tabular}
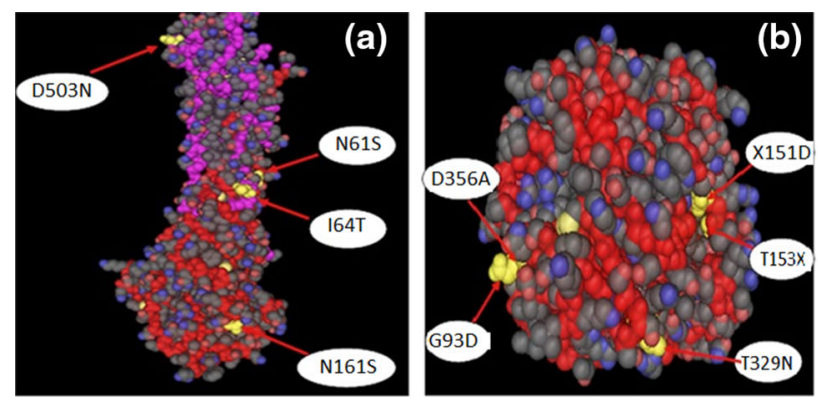

Fig. 2 3D structures of the proteins visualized with Cn3D. a 3D structure of Hemagglutinin, b neuraminidase, $\mathbf{c}$ Matrix protein. Red arrows are showing the mutations in the respective proteins.

shown to contain neuraminidase (NA) substitutions at residues E119V, R292K and N294S in influenza A/H3N2 viruses [9, 11, 26]. Oseltamivir resistance associated mutation at position 119, 292 and 294 of NA was absent in all H3N2 isolates of 2012. Nevertheless, the S31N substitution in the M2 protein indicates resistance to amantadine [17] the influenza matrix ion channel inhibitory drug is present in all Bangladeshi isolates (Fig. 2d).

\section{Discussion}

Characterization of HA, NA and M proteins from all four H3N2 Bangladeshi strains isolated in 2012 showed that the isolates clustered closely with the other global circulates of $\mathrm{A} / \mathrm{H} 3 \mathrm{~N} 2$ during the same time period. The scenario was similar for phylogenetic trees constructed using all four genes. In most cases, Group I showed the clustering of isolates from 2004 to 2012 all over the world including the Bangladeshi isolates used in this study. Other groups consist of isolates from 1968 to before emergence of a newly drifted strain in 2004. These phylogenetic analyses of global isolates provide a comprehensive knowledge of the evolutionary history of A/H3N2 from 1968 to 2012.

The phylogenetic tree of HA gene showed that Bangladeshi isolates clustered with recent strains of America, Asia, Europe and Africa. Moreover, the Bangladeshi isolates of HA genes shows $>99$ similarity with vaccine strains A/Victoria/361/2011. Furthermore, we did not find
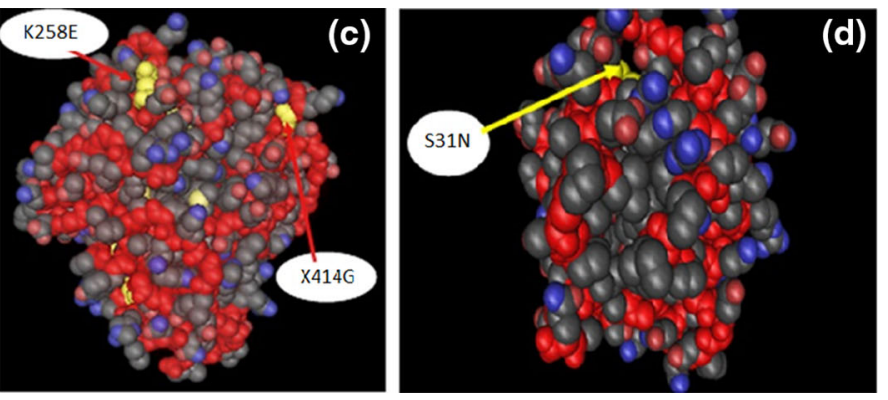

d Structure of M2 channel Matrix protein; yellow arrow shows the mutation on $\mathrm{S} 31 \mathrm{~N}$ which lead the amantadine resistance (color figure online)

any evidence of a significant distinct subclade of viruses circulating in Bangladesh over the period of study. Similar pattern was also observed in the phylogenetic tree of other segments i.e. NA and $\mathrm{M}$.

The existence of $N$-glycosylation is essential for viral membrane glycoproteins and nine potential $N$-linked glycosylation sites were found in Bangladeshi Influenza H3N2 strains from this study similar to Vaccine Strains A/Victoria/ 361/2011 at positions NST024-026, NGT038-040, NCT079-081, NWT142-144, NGT149-151, NVT181-183, NST262-264, NGS301-303 and NGT499-501, respectively. The $\mathrm{N}$-glycans encode required information for folding, maturation, transport or degradation of proteins [19]. Glycosylation seems to be one mechanisms by which a virus masks its epitopes and escapes neutralization by the host antibodies [12, 13]. In addition, some studies have associated increasing glycosylations with reduced virulence $[13,24]$, while others associated the loss of glycosylations with exposure of new receptor binding sites or new decoy epitopes [1].

The M2 is the target of the anti-influenza drugs amantadine and Oseltamivir. Resistance-associated mutation to adamantanes S31N has been detected in all four Bangladeshi $\mathrm{H} 3 \mathrm{~N} 2$ viruses indicating resistance to amantadine. Moreover, Oseltamivir resistance associated mutation at position 119, 292 and 294 of NA was absent in all H3N2 isolates of 2012.

One of the major limitations of this study is relatively small sample size obtained from a single geographical 
origin over a short period of time and the sequencing of only three segments out of eight genes. The data demonstrated that amino acid changes were limited to some key codons at or near antibody-binding sites A-E and variable region along with catalytic and framework site on HA and NA molecule. The changes at the antibody-binding sites or receptor-binding sites are crucial for antigenic drift, and it is important to monitor new $\mathrm{H} 3 \mathrm{~N} 2$ isolates for mutations in these sites. Nevertheless, our findings contribute to a better understanding of the genetic and antigenic evolution of influenza $\mathrm{A} / \mathrm{H} 3 \mathrm{~N} 2$ viruses. Though we have found the recommended vaccine strain for northern hemisphere is highly similar to Bangladeshi isolates, we suggest that a combination of phylogenetic analysis, evaluation of specific mutations in so-called antigenic sites and immunological assays are all important to consider for the optimization of vaccine strain selection.

In many countries the vaccine virus is usually recommended several months prior to the corresponding seasonal changes for vaccine manufacturer to have enough time for effective vaccine development. However, in Bangladesh, although facilities exist for routine serological and detailed genomic characterization of $\mathrm{H} 3 \mathrm{~N} 2$, such studies are not in practice and mostly due to the lack of funding. Phylogenetic analysis shows that viruses are mostly clustered based on their time of isolation rather on geographical locations. Moreover, due to the availability of high-throughput sequencing facilities, whole genome characterization should involve substantially larger number of samples for better understanding of the evolutionary dynamics and route of transmission in different geographical locations as well as effective intervention and vaccine treatment.

Acknowledgements We acknowledge Dhaka Medical College Hospital for providing the samples and the financial support of North South University.

\section{References}

1. Alymova IV, Taylor G, Mishin VP, Watanabe M, Murti KG, Boyd $\mathrm{K}$, et al. Loss of the $N$-linked glycan at residue 173 of human parainfluenza virus type 1 hemagglutinin-neuraminidase exposes a second receptor-binding site. J Virol. 2008;82:8400-10.

2. Blick TJ, Sahasrabudhe A, McDonald M, Owens IJ, Morley PJ, Fenton RJ, McKimm-Breschkin JL. The interaction of neuraminidase and hemagglutinin mutations in influenza virus in resistance to 4-guanidino-Neu5Ac2en. Virology. 1998;246:95-103.

3. Chutinimitkul S, Chieochansin T, Payungporn S, Samransamruajkit R, Hiranras T, Theamboonlers A, et al. Molecular characterization and phylogenetic analysis of $\mathrm{H} 1 \mathrm{~N} 1$ and $\mathrm{H} 3 \mathrm{~N} 2$ human influenza A viruses among infants and children in Thailand. Virus Res. 2008;132:122-31.

4. Clarke LM, Alexander H, Baker MB, Bankowski MJ, Kirk C, et al. Viral culture; approved guideline. CLSI document M41-A. In: Wilhelm DM, editor. Viral culture; approved guideline CLSI document M41-A. Wayne: Clinical And Laboratory Standards Institute; 2003.

5. Colman PM, Hoyne PA, Lawrence MC. Sequence and structure alignment of paramyxovirus hemagglutinin-neuraminidase with influenza virus neuraminidase. J Virol. 1993;67:2972-80.

6. Daum LT, Shaw MW, Klimov AI, Canas LC, Macias EA, Niemeyer D, et al. Influenza A (H3N2) outbreak, Nepal. Emerg Infect Dis. 2005;11:1186-91.

7. Gulati U, Hwang C-C, Venkatramani L, Gulati S, Stray SJ, Lee JT, Laver WG, Bochkarev A, Zlotnick A, Air GM. Antibody epitopes on the neuraminidase of a recent $\mathrm{H} 3 \mathrm{~N} 2$ influenza virus (A/Memphis/31/98). J Virol. 2002;76:12274-80.

8. Hungnes O. Early detection of A/California/7/2004-like A (H3N2) strains in Norway during the 2004-2005 season, and emergence of a novel $\mathrm{H} 3 \mathrm{~N} 2$ genetic sub-variant toward the end. Vaccine. 2006;24(44):6694-6.

9. Ison MG, Hayden FG. Viral infections in immunocompromised patients: what's new with respiratory viruses? Curr Opin Infect Dis. 2002; 15:355-67.

10. Kimura M. A simple method for estimating evolutionary rates of base substitutions through comparative studies of nucleotide sequences. J Mol Evol. 1980;16:111-20.

11. Kiso M, Mitamura K, Sakai-Tagawa Y, Shiraishi K, Kawakami C, Kimura K, Hayden FG, Sugaya N, Kawaoka Y. Resistant influenza A viruses in children treated with oseltamivir: descriptive study. Lancet. 2004;364:759-65.

12. Long J, Bushnell RV, Tobin JK, Pan K, Deem MW, Nara PL, et al. Evolution of $\mathrm{H} 3 \mathrm{~N} 2$ influenza virus in a Guinea pig model. PLoS ONE. 2011;6:e22844.

13. Mishin VP, Novikov D, Hayden FG, Gubareva LV. Effect of hemagglutinin glycosylation on influenza virus susceptibility to neuraminidase inhibitors. J Virol. 2005;79:12416-24.

14. Palese P, Shaw ML. Orthomyxoviridae Viruses Their Replication. In: Knipe DM, Howley PM, editors. Fields Virology. Philadelphia, PA: Lippincott Williams Wilkins, Alphen aan den Rijn: Wolters Kluwer Bus; 2007. 1647-89.

15. Pechirra P, Gonçalves P, Arraiolos A, Coelho A, Rebelo-deAndrade H. Characterization of influenza A/Fujian/411/2002 (H3N2)-like viruses isolated in Portugal between 2003 and 2005. J Med Virol. 2008;80(9):1624-30.

16. Reid AH, Taubenberger JK, The Fanning TG. Spanish influenza: integrating history and biology. Microbes Infect. 1918;2001:81-7.

17. Saito R, Sakai T, Sato I, Sano Y, Oshitani H, Sato M, Suzuki H. Frequency of amantadine-resistant influenza A viruses during two seasons featuring cocirculation of $\mathrm{H} 1 \mathrm{~N} 1$ and $\mathrm{H} 3 \mathrm{~N} 2$. J Clin Microbiol. 2003;41:2164-5.

18. Saitou N, Nei M. The neighbor-joining method: a new method for reconstructing phylogenetic trees. Mol Biol Evol. 1987;4:406-25.

19. Sun S, Wang Q, Zhao F, Chen W, Li Z. Glycosylation site alteration in the evolution of influenza a $(\mathrm{H} 1 \mathrm{~N} 1)$ viruses. PLoS ONE. 2011;6:e22844.

20. Tamura K, Peterson D, Peterson N, Stecher G, Nei M, Kumar S. MEGA5: molecular evolutionary genetics analysis using maximum likelihood, evolutionary distance, and maximum parsimony methods. Mol Biol Evol. 2011;28:2731-9.

21. Tomar AK, Sooch BS, Yadav S. Computational analysis of Concanavalin A binding glycoproteins of human seminal plasma. Bioinformation. 2011;7:69-75.

22. Tong S, Zhu X, Li Y, Shi M, Zhang J, Bourgeois M, Bourgeois M, Yang H, Chen X, Recuenco S, Gomez J, Chen LM, Johnson A, Tao Y, Dreyfus C, Yu W, McBride R, Carney PJ, Gilbert AT, Chang J, Guo Z, Davis CT, Paulson JC, Stevens J, Rupprecht CE, Holmes EC, Wilson IA, Donis RO. New world bats harbor diverse influenza a viruses. PLoS Pathog. 2013;9:e1003657. 
23. Underwood PA. Mapping of antigenic changes in the haemagglutinin of Hong Kong influenza (H3N2) strains using a large panel of monoclonal antibodies. J Gen Virol. 1982;62:153-69.

24. Vigerust DJ, Ulett KB, Boyd KL, Madsen J, Hawgood S, Mccullers JA. $N$-linked glycosylation attenuates H3N2 influenza viruses. J Virol. 2007;81:8593-600.

25. Wang Y, Geer LY, Chappey C, Kans JA, Bryant SH. Cn3D: sequence and structure views for Entrez. Trends Biochem Sci. 2000;25:300-2.
26. Whitley RJ, Hayden FG, Reisinger KS, Young N, Dutkowski R, Ipe D, Mills RG, Ward P. Oral oseltamivir treatment of influenza in children. Pediatr Infect Dis J. 2001;20:127-33.

27. Wiley DC, Wilson IA, Skehel JJ. Structural identification of the antibody-binding sites of Hong Kong influenza haemagglutinin and their involvement in antigenic variation. Nature. $1981 ; 289: 373-8$. 